\title{
Small RNA derived from Tobacco mosaic virus targets a host C2-domain abscisic acid-related (CAR) 7-like protein gene
}

\author{
Song Guo ${ }^{1}$ and Sek-Man Wong ${ }^{1,2,3^{*}}$ (D)
}

\begin{abstract}
Tobacco mosaic virus (TMV) is a positive-sense single-stranded RNA virus. The $3^{\prime}$ end of TMV genome is consisted of an upstream pseudoknot domain (UPD) and a tRNA-like structure (TLS), both of which are important RNA elements to enhance TMV replication and translation. Deep-sequencing analysis revealed that TMV-specific viral small interfering RNAs (vsiRNAs) were generated in TMV-infected Nicotiana benthamiana plants. A vsiRNA derived from the juxtaposition between UPD and TLS, named TMV-vsiRNA 22 nt (6285-6306), possessed high sequence complementarity to a host gene which encodes a C2-domain abscisic acid (ABA)-related (CAR) 7-like protein. CAR proteins play a critical role in ABA signaling pathway. The CAR protein-encoding gene was amplified from $N$. benthamiana leaves and termed as Nb-CAR7. In TMV-infected plants, accumulation of Nb-CAR7 transcripts was significantly decreased, as compared with that of mock-inoculated and TMV-43A-infected plants. TMV-43A is a mutant without the UPD sequence in its genome. Overexpression of Nb-CAR7 led to decreased TMV RNA accumulation in the TMV-inoculated leaves. Silencing of Nb-CAR7 enhanced TMV replication and resulted in a higher viral RNA accumulation. In addition, the expression level of Nb-CAR7 was positively correlated to that of a low-temperature-induced ABA responsive gene (LTI65). The effect of Nb-CAR7 on TMV RNA accumulation in host plants was linked to ABA signaling pathway. In conclusion, a vsiRNA derived from the juxtaposition between UPD and TLS at the $3^{\prime} U T R$ of TMV targets a host CAR7 gene.
\end{abstract}

Keywords: Tobacco mosaic virus, Virus small interfering RNA, C2 domain ABA-related (CAR) gene

\section{Background}

Tobacco mosaic virus (TMV) is a positive-sense singlestranded RNA virus in genus Tobamovirus. It has a wide host range and its infection can cause severe damages and yield losses in many economically important crops (Scholthof et al. 2011). In its natural host tobacco and other closely related species, TMV can induce chlorosis, mosaic and necrosis in plant tissues. The virus is mechanically transmitted, resulting in quick and effective infection (Scholthof 2004). Discovery of new molecular interactions

\footnotetext{
* Correspondence: dbswsm@nus.edu.sg

'Department of Biological Sciences, National University of Singapore, Singapore 117543, Singapore

${ }^{2}$ Temasek Life Sciences Laboratory, 1 Research Link, Singapore 117604, Singapore

Full list of author information is available at the end of the article
}

between TMV and its host proteins will add to our knowledge to connect missing links and help in developing control strategies to prevent spread of the virus.

The RNA genome of TMV contains four open reading frames that encode essential viral proteins for replication, movement and encapsidation. There are also several elements involved in control of replication and translation of viral genes in the $5^{\prime}$ and $3^{\prime}$ untranslated regions (UTRs). For example, the upstream pseudoknot domain (UPD) and tRNA-like structure (TLS) in the 3' UTR play important roles in virus replication (Gallie and Walbot 1990; Takamatsu et al. 1990). The TLS of TMV can be aminoacylated and some primary RNA elements in UPD interact with host plant elongation factors during virus replication (Gallie et al. 1991; Osman et al.

(c) The Author(s). 2020 Open Access This article is licensed under a Creative Commons Attribution 4.0 International License, which permits use, sharing, adaptation, distribution and reproduction in any medium or format, as long as you give

appropriate credit to the original author(s) and the source, provide a link to the Creative Commons licence, and indicate if changes were made. The images or other third party material in this article are included in the article's Creative Commons licence, unless indicated otherwise in a credit line to the material. If material is not included in the article's Creative Commons licence and your intended use is not permitted by statutory regulation or exceeds the permitted use, you will need to obtain permission directly from the copyright holder. To view a copy of this licence, visit http://creativecommons.org/licenses/by/4.0/. 
2000; Zeenko et al. 2002). A novel stem-loop structure located upstream of the UPD of TMV has been identified to regulate virus replication. Disruption of this stem-loop structure enhances TMV infectivity and viral RNA accumulation (Guo and Wong 2018).

RNA silencing is an evolutionarily conserved mechanism in eukaryotes. It is a defense mechanism induced by doublestranded RNAs (dsRNAs) or hairpin structured RNA (hpRNA) that leads to degradation of target RNAs (Zhang et al. 2019). In plants, RNA silencing acts as a defense system against infection by pathogens, including fungi, bacteria, viruses or viroids, which results in the production of pathogen-specific short interfering RNAs (siRNAs) (Guo et al. 2016). In virus-infected plants, virus-derived small interfering RNAs (vsiRNAs) are produced from doublestranded viral RNA by host plant enzymes. The vsiRNAs are loaded into Argonaute (AGO)-containing complexes known as RNA-induced silencing complexes (RISCs), to promote the degradation of both genomic and subgenomic viral RNAs in a sequence-specific manner (Eamens et al. 2008).

The accumulation of vsiRNAs is an indication of the presence of RNA silencing in plants after virus infection (Li et al. 2018). In order to overcome the host antiviral RNA silencing system, plant viruses encode RNAi-suppressors and use them to counter the host RNA-factors and to weaken host defense. Most suppressors bind vsiRNAs to prevent degradation of viral RNAs so as to inhibit RNA silencing effects (Bivalkar-Mehla et al. 2011). Some suppressors interact with host genes to compromise antiviral functions of these genes (Li and Wang 2018). Moreover, post-transcriptional gene silencing is compromised in the presence of viral RNAi suppressors, which leads to over-expression of host defenserelated genes to protect the host against viral infection (Pumplin and Voinnet 2013). For example, in Sugarcane mosaic virus (SCMV)-infected maize, the binding of RNAisuppressor of SCMV (HC-Pro) with the violaxanthin deep oxidase protein of maize (ZmVDE) down-regulates the RNA silencing suppression activity of HC-Pro and results in reduced accumulation of viral RNA and coat protein in the infected cells (Chen et al. 2017).

A chimeric virus TMV-43A was created previously (Niu et al. 2015). In TMV-43A genome, the original TMV-UPD sequence is deleted and substituted by a 43nt poly(A) tract. TMV-43A induces mosaic symptoms in $N$. benthamiana and replicates slower than TMV (Niu et al. 2015). Comparing with the vsiRNAs generated in TMV-43A-infected plants, many TMV UPD-specific vsiRNAs were generated in TMV-infected plants. Either partial or entire sequences of these TMV-specific vsiRNAs were all mapped to TMV-UPD. These vsiRNAs are predicted to target a number of host genes involved in host responses and various signaling pathways (Guo and Wong 2017). Since TMV-43A does not contain the UPD sequence, it can't generate vsiRNAs with UPD sequence.
Abscisic acid (ABA) is a key hormone involved in plant responses to abiotic stresses and has great impacts on plant defense against various pathogens (Alazem and Lin 2015). For example, $\mathrm{ABA}$ induces different plant resistance responses to viruses to interfere with virus accumulation (Alazem and Lin 2017); ABA induces callose deposition at plasmodesmata, a mechanism that limits viral cell-to-cell movement and reduces virus spread throughout the whole plant (Mauch-Mani and Mauch 2005). A number of proteins are involved in ABA biosynthesis and signaling pathway in plants. The ten-member family of C2-domain ABA-related (CAR) proteins in Arabidopsis thaliana consists of single $\mathrm{C} 2$ domains that interact with pyrabactin resistance 1/PYR1-like (PYR/PYL) ABA receptors (Rodriguez et al. 2014). Structural studies revealed that CAR proteins have two lipid binding sites which are calcium $\left(\mathrm{Ca}^{2+}\right)$ sensors with a basal phospholipid binding activity. Clusters of CAR proteins act as a membrane assembly site for ABA receptors through $\mathrm{Ca}^{2+}$-dependent recruitment. This process is relevant to $\mathrm{ABA}$ signaling and CARs represent a signaling node that connects the $\mathrm{ABA}$ and $\mathrm{Ca}^{2+}$ pathways to the membrane (Diaz et al. 2016).

In this study, we discovered a vsiRNA derived from the juxtaposition of TMV-UPD and TLS. It has near-perfect complementarity with the CAR 7-like protein-encoding gene in $N$. benthamiana plants, termed as $N b$-CAR7. This vsiRNA directly silenced the $N b-C A R 7$ gene during viral infection. Accompanying with a high accumulation of TMV-vsiRNA in plants, the expression level of $\mathrm{Nb}$-CAR7 was significantly decreased in TMV-infected plants, as compared to that of mock-inoculated and TMV-43Ainfected plants. Transient overexpression of $\mathrm{Nb}$-CAR7 inhibited TMV infection and resulted in reduced amount of viral RNAs in infiltrated leaves. Silencing of $\mathrm{Nb}$-CAR7 in $N$. benthamiana plants enhanced TMV replication and led to a higher accumulation of viral RNAs. Moreover, the expression of $\mathrm{Nb}$-CAR7 was correlated to that of a lowtemperature-induced $65 \mathrm{KDa}$ protein (LTI65) gene and also an ABA marker gene. Therefore, it is deduced that the effect of $\mathrm{Nb}-\mathrm{CAR7}$ on TMV RNA accumulation was linked to ABA signaling pathway. In summary, our findings showed that a vsiRNA derived from the juxtaposition between UPD and TLS in the 3' UTR of TMV targets a host CAR7 gene in $N$. benthamiana plants.

\section{Results}

vsiRNA derived from the UPD region of TMV genome was detected in TMV-infected $N$. benthamiana plants

Based on our previously published results on small RNA sequencing of TMV-infected $N$. benthamiana plants, a total of 32,369 siRNA reads were mapped to TMV genome (Guo and Wong 2017). The vsiRNAs sequences (either partial or full) mapped to TMV-UPD were selected for analysis. The vsiRNA generated from nucleotides (nt) 6285 to 6306 in TMV positive-sense strand showed the 
highest read counts and was termed as TMV-vsiRNA 22 nt (6285-6306) (Fig. 1a). As the TMV-UPD region expands from 6192 to $6288 \mathrm{nt}$, TMV-vsiRNA $22 \mathrm{nt}$ (62856306) was mapped to the juxtaposition encompassing the UPD and TLS (Fig. 1b). As TMV-43A does not contain the UPD sequence, the TMV-vsiRNA 22 nt (6285-6306) is not generated in TMV-43A infected plants.

The presence of TMV-vsiRNA $22 \mathrm{nt}$ (6285-6306) was validated through Northern blot. High accumulation of TMV-vsiRNA $22 \mathrm{nt}$ (6285-6306) was detected in TMVinfected $N$. benthamiana plants at 4 days post inoculation (dpi) (Fig. 1c). We compared TMV-vsiRNA 22 nt (62856306) sequence with small RNAs generated from TMV43A-infected N. benthamiana (Guo and Wong 2017) and found no match between them. This is due to the reason that TMV-43A does not possess the UPD sequence in its viral genome.

Validation of the putative target gene of TMV-vsiRNA 22 nt (6285-6306) encoding a C2-domain ABA-related 7-like protein in $N$. benthamiana plants

To identify the potential target genes of TMV-vsiRNA $22 \mathrm{nt}$ (6285-6306) in host plant, the TMV-vsiRNA 22 nt (6285-
6306) sequence was aligned to $N$. benthamiana draft genome sequence using an online plant small RNA target analysis tool psRNATarget (Dai et al. 2018). A transcript sequence located in $N$. benthamiana genome scaffold Niben101Scf12415g00017.1 showed near-perfect sequence complementarity with TMV-vsiRNA $22 \mathrm{nt}$ (6285-6306) (Fig. 2a). The BLASTn results showed that this transcript shared 97\% (491/504) sequence identity with a predicted $N$. benthamiana C2-domian ABA-related 7-like gene termed as $\mathrm{Nb}$-CAR7. The accumulation of $\mathrm{Nb}$-CAR7 transcript in TMV-infected $N$. benthamiana plants was significantly lower than that in mock-inoculated plants at 4 dpi. The expression level of $\mathrm{Nb}$-CAR7 in the TMV-43A-infected plants remained the same as that in the mock-inoculated plants (Fig. 2b).

The mRNA cleavage of $\mathrm{Nb}$-CAR7 by TMV-vsiRNA $22 \mathrm{nt}$ (6285-6306) was validated through 5'-RNAlinker-mediated RACE (5'-RLM-RACE). A target DNA fragment was successfully amplified from TMVinfected $N$. benthamiana leaves, but this product was not amplified from mock-inoculated plants (Fig. 2c), indicating the specificity of TMV-vsiRNA $22 \mathrm{nt}$-directed cleavage of the $\mathrm{Nb}$-CAR7 transcript in TMVinfected $N$. benthamiana plants.

\section{a}

\begin{tabular}{lccccr}
\hline \multicolumn{1}{c}{ Sequence (5'-3') } & Length & Start & Stop & \multicolumn{2}{c}{ Strand Read Counts } \\
\hline TCGAAGGGTTGTGTCTTGGATCGCG & 25 & 6282 & 6306 & + & 10 \\
CGAAGGGTGTGTCTTGGATCGCG & 24 & 6283 & 6306 & + & 77 \\
GAAGGGTGTGTCTTGGATCGCG & 23 & 6284 & 6306 & + & 157 \\
\hline AAGGGTGTGTCTTGGATCGCG & 22 & 6285 & 6306 & + & 9787 \\
\hline CGAAGGGTTGTTCTTGGATCGCGC & 25 & 6283 & 6307 & + & 237 \\
GAAGGGTGTGTCTTGGATCGCGC & 24 & 6284 & 6307 & + & 349 \\
AAGGGTGTGTCTTGGATCGCGC & 23 & 6285 & 6307 & + & 468 \\
GAAGGGTGTGTCTTGGATCGCGCG & 25 & 6284 & 6308 & + & 223 \\
AAGGGTTGTGTTTGGATCGCGCG & 24 & 6285 & 6308 & + & 790 \\
AAGGGTGTGTCTTGGATCGCGCGG & 25 & 6285 & 6309 & + & 489 \\
\hline
\end{tabular}

b

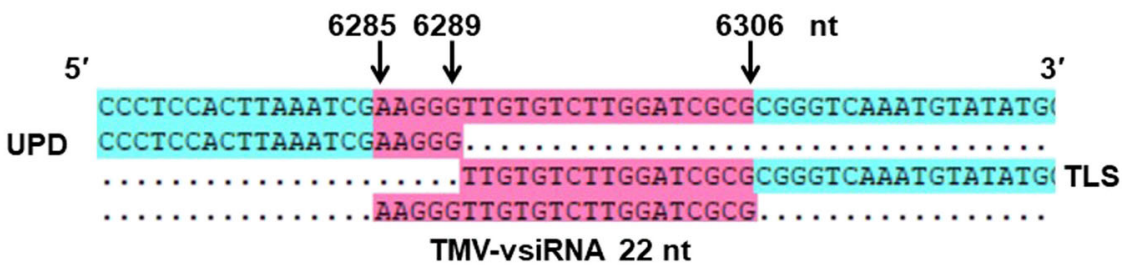

C

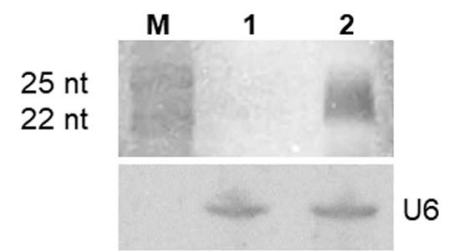

M: Marker

1: Mock

2: TMV-infected

Fig. 1 vsiRNA derived from the UPD region of TMV genome was detected in TMV-infected N. benthamiana plants. a vsiRNA generated from nt 6285 to nt 6306 in TMV positive-sense strand was termed as TMV-vsiRNA 22 nt (6285-6306). It showed the highest read counts of 9787 (read box) in all TMV-UPD-vsiRNAs. $\mathbf{b}$ The exact nucleotide positions of TMV-vsiRNA $22 \mathrm{nt}$ (6285-6306) sequence, which was mapped to the juxtaposition of UPD and TLS. c Northern blot analysis showed that TMV-vsiRNA 22 nt (6285-6306) was detected in TMV-infected N. benthamiana plants at 4 dpi but not in mock plants. U6 stands for U6 snRNA used as loading control 


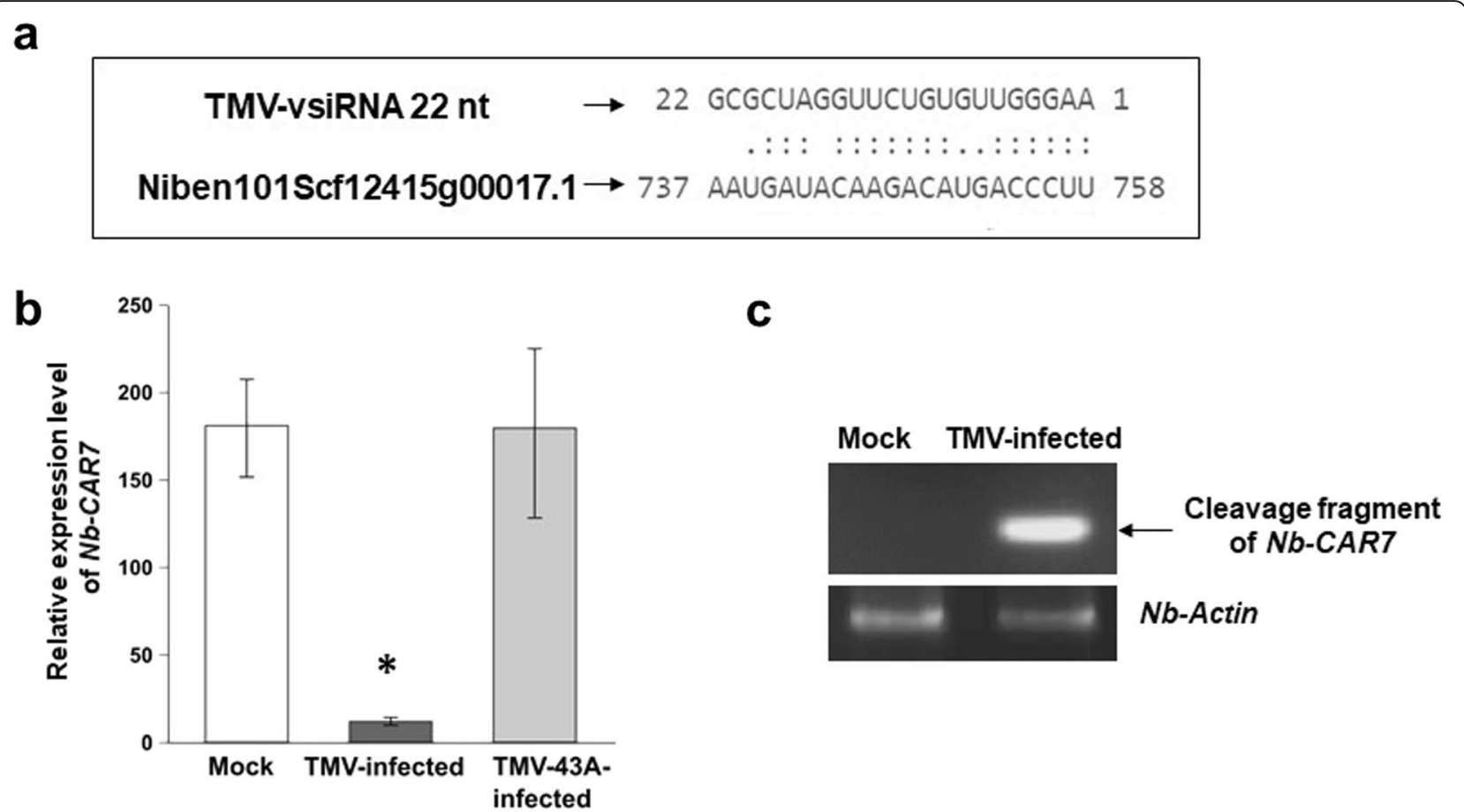

Fig. 2 TMV-vsiRNA 22 nt (6285-6306) targets a C2-domain ABA-related 7-like protein in N. benthamiana plants after virus infection. a Prediction of a putative host target gene of TMV-vsiRNA 22 nt (6285-6306). A transcript sequence located in N. benthamiana genome scaffold

Niben101Scf12415g00017.1 showed near-perfect sequence complementarity with TMV-vsiRNA $22 \mathrm{nt}$ (6285-6306). b The relative expression level of $\mathrm{Nb}$-CAR7 in TMV-infected plants was lower than that in mock-inoculated and TMV-43A-infected N. benthamiana plants at 4 dpi. * represents $P<0.05$ compared with mock-inoculated group. c A unique Nb-CAR7 fragment was amplified by 5'-RLM-RACE from the TMV-infected $N$. benthamiana plants but not from the mock-inoculated plants, indicating TMV-vsiRNA 22 nt-directed cleavage of the Nb-CAR7 transcript

Transient overexpression of $\mathrm{Nb}-\mathrm{CAR7}$ inhibited TMV RNA accumulation in $N$. benthamiana plants

To examine the effect of $\mathrm{Nb}-\mathrm{CAR7}$ on TMV infection, $\mathrm{Nb}$-CAR7 overexpression construct driven by the $35 \mathrm{~S}$ promoter with GFP tag was agro-infiltrated into $N$. benthamiana leaves (Fig. 3a). At 3 days after infiltration, the expression level of $\mathrm{Nb}-\mathrm{CAR} 7$ was significantly increased in the 35S-NbCAR7-GFP-infiltrated leaves, as compared to that of GFP-expressed control plants (Fig. 3b). After TMV was inoculated onto the infiltrated leaves, TMV RNA accumulation was significantly reduced in the inoculated leaves of $\mathrm{Nb}$-CAR7-overexpressed plants at $7 \mathrm{dpi}$, whereas was higher in the upper leaves of the same plant, as compared with that of the control plants (Fig. 3c).

After TMV inoculation, the transcript levels of $\mathrm{Nb}$-CAR7 in the inoculated leaves and upper leaves of infiltrated plants at $7 \mathrm{dpi}$ were also analysed (Fig. 3d). The transcript level of $\mathrm{Nb}-\mathrm{CAR7}$ in the TMV-inoculated leaves was higher than that of control plants. Conversely, $\mathrm{Nb}$-CAR7 level in the upper leaves was lower than that of control.

Silencing of $\mathrm{Nb}$-CAR7 enhanced TMV RNA accumulation in $N$. benthamiana plants

To examine the roles of $\mathrm{Nb}-\mathrm{CAR7}$ in TMV infection, $\mathrm{N}$. benthamiana plants were infiltrated first with a Tobacco rattle virus (TRV) vector containing partial $\mathrm{Nb}-\mathrm{CAR7}$ sequence (101-497 nt) (TRV2:Nb-CAR7) to silence the expression of $\mathrm{Nb}$-CAR7. At 7 days post infiltration, transcription of $\mathrm{Nb}-\mathrm{CAR} 7$ was not detected in the infiltrated leaves, indicating that silencing of $\mathrm{Nb}-\mathrm{CAR} 7 \mathrm{had}$ occurred. The transcript level of $\mathrm{Nb}-\mathrm{CAR7}$ decreased in the upper leaves of silenced plants, as compared to that of TRV2-infiltrated control plants (Fig. 4a). While the expression of $\mathrm{Nb}-\mathrm{CAR7}$ was not detected after TRVinduced silencing, TMV RNA accumulation was increased in both the TMV-inoculated and upper leaves of the $N b$-CAR7-silenced plants (Fig. 4b). These results suggested that silencing of $N b-C A R 7$ enhanced TMV RNA accumulation in $N$. benthamiana plants.

Effect of $\mathrm{Nb}$-CAR7 on TMV RNA accumulation was linked to $A B A$ signaling pathway

CAR proteins play important roles in plant ABA signaling pathway. LTI65 is an ABA marker gene with an ABA-induced cis-acting element in its promotor region (Yamaguchi-Shinozaki and Shinozaki 1994). Our results showed that the relative expression level of $\mathrm{Nb}$-LTI65 was up-regulated in transiently $\mathrm{Nb}$-CAR7-overexpressed leaves of $N$. benthamiana plants after TMV infection (Fig. 5a) but was down-regulated in $N b$-CAR7-silenced 
a
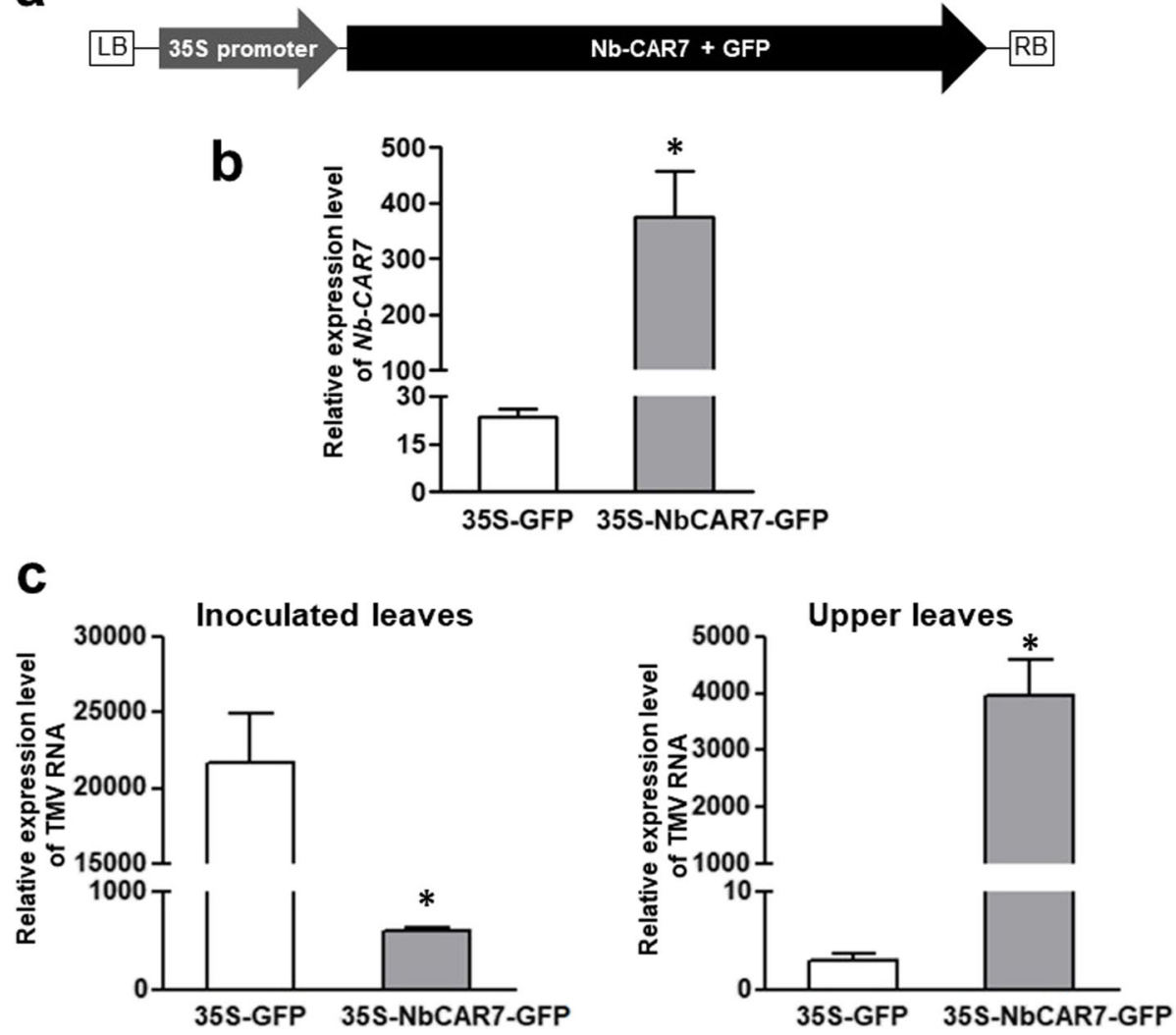

d
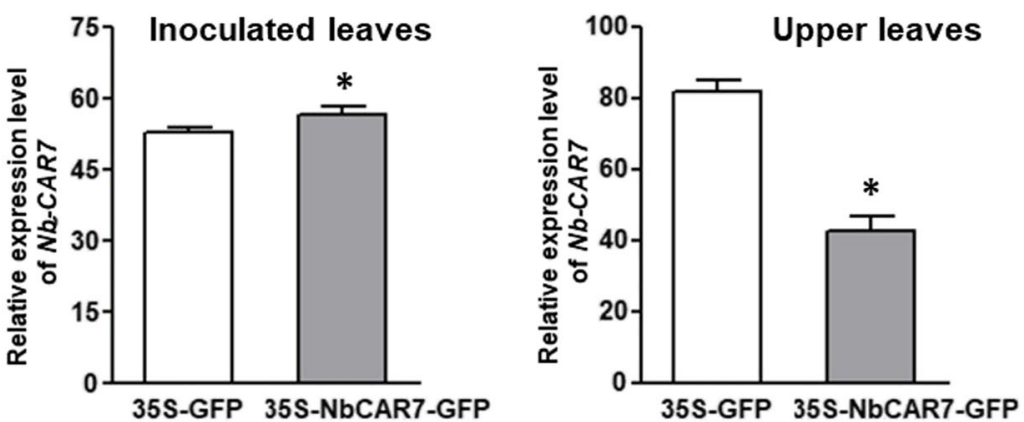

Fig. 3 Transient overexpression of Nb-CAR7 inhibited TMV RNA accumulation in N. benthamiana plants. a Schematic structure of Nb-CAR7 overexpression vector, 35S-NbCAR7-GFP. b The relative expression level of Nb-CAR7 was increased in plant leaves at 3 days post infiltration with 35S-NbCAR7-GFP vector, using plants infiltrated with 35S-GFP vector as controls. c After TMV inoculation of the plants pre-infiltrated with 35SNbCAR7-GFP vector, the relative expression level of TMV RNA at 7 dpi was decreased in the TMV-inoculated leaves but increased in the upper leaves. $\mathbf{d}$ After TMV inoculation of the plants pre-infiltrated with 35S-NbCAR7-GFP vector, the relative expression level of Nb-CAR7 in the TMVinoculated leaves was higher than that of control plants at $7 \mathrm{dpi}$, whereas was lower in the upper leaves than that of control plants. ${ }^{*}$ represents $P<0.05$ compared with control group

plants (Fig. 5b). Therefore, the effect of $N b$-CAR7 on TMV RNA accumulation was linked to the ABA signaling pathway.

\section{Discussion}

Small RNA-mediated silencing is a widespread antiviral mechanism in plants and other organisms. Many viruses encode suppressors of RNA silencing to counter defense.
The p126 protein of TMV is an RNA silencing suppressor and multiple domains of p126 protein can independently suppress local and systemic RNA silencing (Wang et al. 2012). The vsiRNAs have been reported to target host genes to mediate disease symptoms in plants. Those generated from $\mathrm{Cu}$ cumber mosaic virus (CMV) Y-satellite (Y-sat) RNA directed gene silencing of host chlorophyll biosynthesis-related gene and induced chlorotic symptom in plants after virus infection 


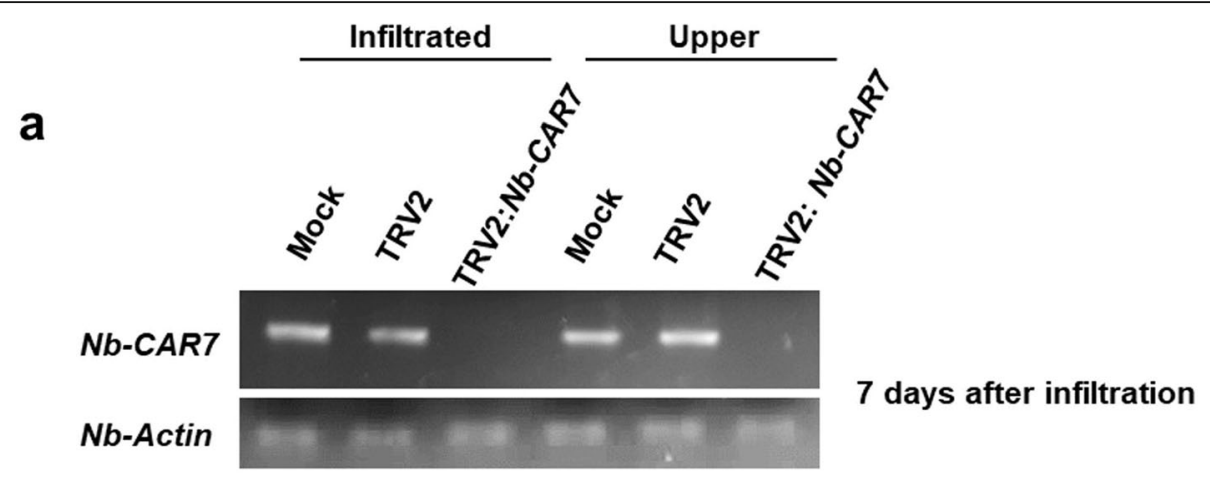

b

Inoculated leaves

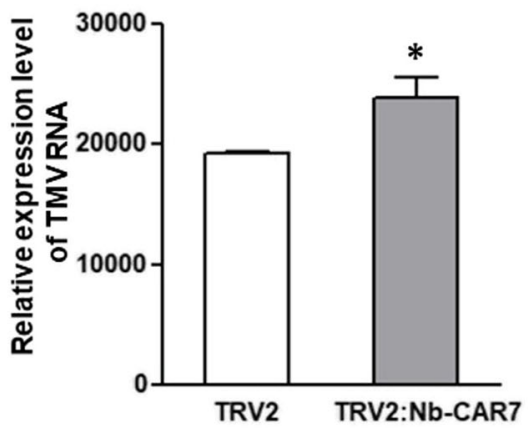

Upper leaves

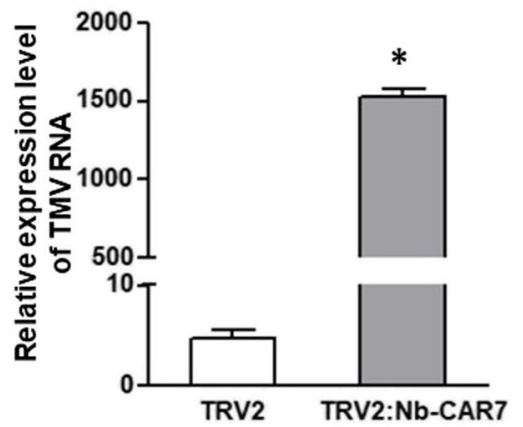

Fig. 4 Silencing of Nb-CAR7 enhanced TMV RNA accumulation in N. benthamiana plants. a The Nb-CAR7 transcript was not detected in both the infiltrated leaves and upper leaves of the TRV2:Nb-CAR7-infiltrated plants at 7 days post infiltration. As a control, the Nb-Actin was detected in the same plants at the same time point. b Relative accumulation of TMV RNA was increased in both TMV-inoculated and upper leaves of the NbCAR7-silenced plants. * represents $P<0.05$ compared with control group

(Shimura et al. 2011). In this study, the TMV-derived vsiRNAs showed sequence complementarity to the host gene $N b$ CAR7 that belongs to the ten-member family of CAR proteins in plants. CAR proteins act as a membrane assembly site for ABA receptors (Diaz et al. 2016). Transient calciumdependent interactions of PYR/PYL ABA receptors with membranes are meditated through CAR proteins, indicating the importance of CAR proteins in early ABA signaling processes (Rodriguez et al. 2014). After TMV infection, the accumulation of TMV-vsiRNA $22 \mathrm{nt}$ (6285-6306) resulted in silencing of the $\mathrm{Nb}-\mathrm{CAR7}$ gene. When $\mathrm{Nb}$-CAR7 was overexpressed, TMV RNA accumulation was reduced in host plants. On the other hand, when $N b$-CAR7 was silenced in plants, TMV RNA accumulation increased. These results showed a negative correlation between transcript abundance of $\mathrm{Nb}$ CAR7 and TMV RNA accumulation.

All CAR proteins contain the $\mathrm{C} 2$ domain, which is a $\mathrm{Ca}^{2+}$-dependent membrane-targeting module present in many cellular proteins that are involved in signal transduction or membrane trafficking. The unique feature of C2 domain is that it has a wide range of lipid selectivity for major components of cell membranes. In addition, it is involved in $\mathrm{Ca}^{2+}$-dependent phospholipid binding and in membrane targeting which guides lipids to the correct locations of ABA receptors (Stahelin et al. 2003; Corbin et al. 2007; Rodriguez et al. 2014; Diaz et al. 2016). Therefore, CAR proteins play a critical role in the ABA signaling process.

The nucleotide sequence of $\mathrm{Nb}$-CAR7 was compared with those of other CAR7 genes in tobacco plants and the result (Fig. 6) showed that CAR7 genes are conserved among the tobacco plants, and these genes showed a high similarity with each other. The TMV-vsiRNA 22 nt (6285-6306) would also be able to target CAR7 genes in other tobacco plants, to counterwork host defense responses after virus infection.

The expression of LTI65 is induced in response to water deprivation conditions such as cold, high salt, and desiccation. The responses are mediated through ABAdependent signaling pathway (Yamaguchi-Shinozaki and Shinozaki 1993; Uno et al. 2000; Nakashima et al. 2006). In this study, $\mathrm{Nb}-L T I 65$ was amplified and used as a marker gene to monitor the level of ABA expression in transiently $N b$-CAR7-overexpressed leaves or TRVinduced-Nb-CAR7-silenced plants after TMV infection. The transcript level of $N b$-LTI65 was increased in $N b$ CAR7-overexpressed leaves, and decreased in $\mathrm{Nb}$-CAR7silenced plants, suggesting that $\mathrm{ABA}$ expression varied in accordance with the amount of $N b$-CAR7 in plants. Our results showed a positive correlation between the expression of $\mathrm{Nb}-\mathrm{CAR} 7$ and $\mathrm{Nb}$-LTI65. However, there 

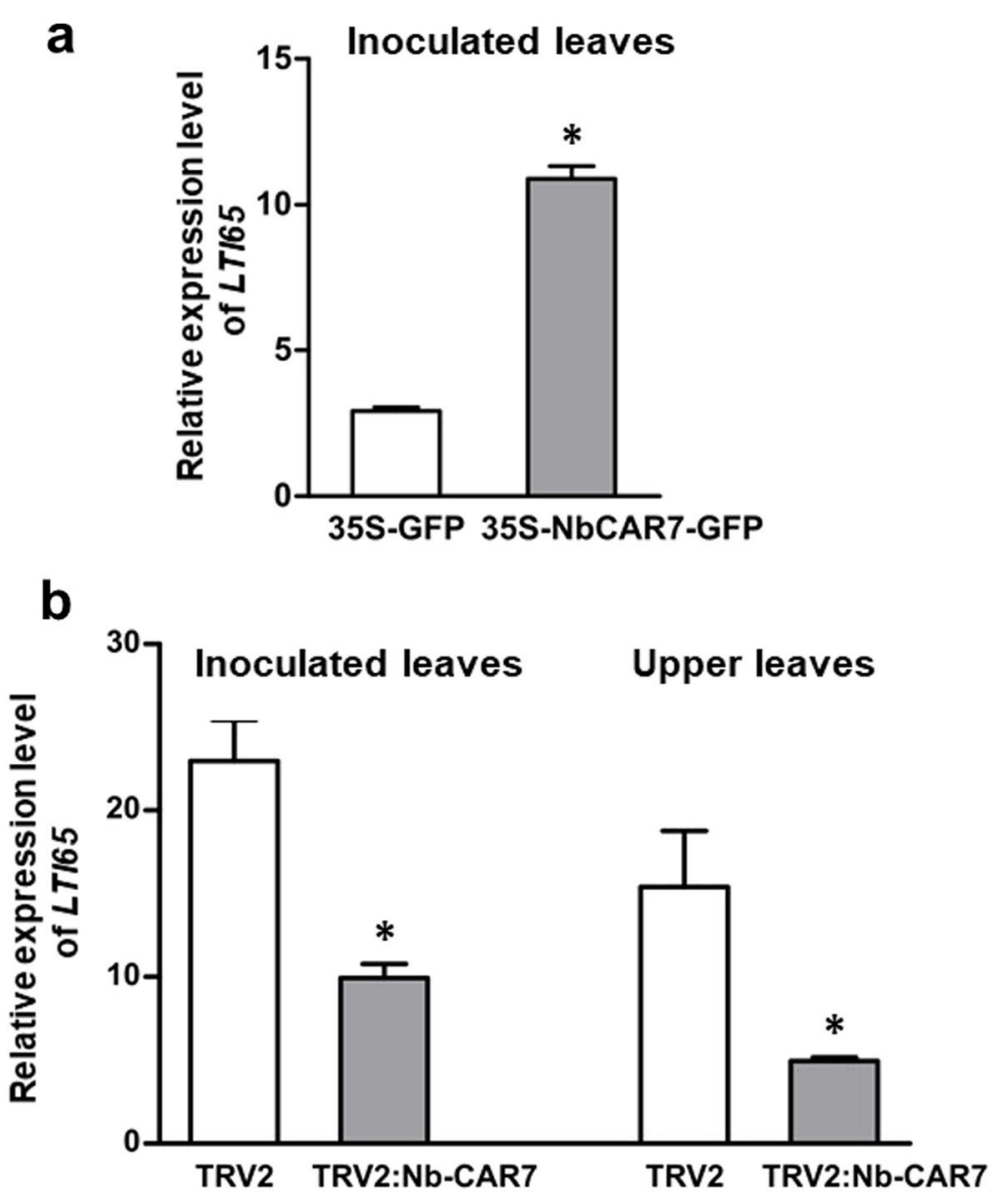

Fig. 5 Effect of Nb-CAR7 on TMV RNA accumulation was linked to ABA signaling pathway. a Relative expression level of Nb-LT165 was upregulated in the inoculated leaves of $\mathrm{Nb}$-CAR7-overexpressed plants, but $\mathbf{b}$ down-regulated in both inoculated and upper leaves of $\mathrm{Nb}$-CAR7silenced plants. ${ }^{*}$ represents $P<0.05$ compared with control group

is a negative correlation between the expression of $\mathrm{Nb}$ CAR7 and accumulation of TMV RNA. As CAR proteins are involved in ABA signaling, our results showed that the effect of $\mathrm{Nb}$-CAR7 on TMV RNA accumulation was linked to the ABA signaling pathway. Further tests will be carried out to determine whether or not the downregulation of $\mathrm{Nb}$-CAR7 is mediated by TMV-siRNA 22 nt (6285-6306).

The overexpression of $\mathrm{Nb}$-CAR7 through agrobacterium infiltration was restricted to plant cells in the infiltrated region. Therefore, TMV RNA accumulation was decreased only in the infiltrated leaves but not in the upper leaves after TMV was inoculated into the agrobacterium-infiltrated leaves. (Fig. 3c). RNA silencing of $\mathrm{Nb}$-CAR7 occurred in the upper leaves due to transient overexpression of $\mathrm{Nb}-\mathrm{CAR7}$ in the infiltrated leaves, which led to a lower amount of $N b-C A R 7$ in the upper leaves (Fig. 3d). Therefore, the accumulation of TMV RNA remained high in the upper leaves. In contrast, silencing of $\mathrm{Nb}$-CAR7 through TRV-based and VIGSinduced gene silencing occurred throughout the entire plant. After TMV infection, silencing of $N b-C A R 7$ led to a higher viral RNA accumulation in both inoculated and upper leaves of $N b$-CAR7-silenced plants (Fig. 4b). Our results showed that the $N b-C A R 7$ affects TMV replication. In future work, we will generate $N b$-CAR7-transgenic plants to verify its effects on TMV infection.

ABA is a key plant hormone which has a great impact on plant defense against various pathogens (Alazem and Lin 2015; Sah et al. 2016; Alazem and Lin 2017). ABA enhances plant defense by shutting stomata and inducing callose deposition at cell walls (Ellinger et al. 2013). If a pathogen is successfully established inside a plant tissue, ABA induction can hinder plant defense by antagonizing other hormone pathways (Anderson et al. 2004; Yasuda et al. 2008). Therefore, ABA is labeled as a phase-specific modulator of defense responses. However, ABA induces different resistance mechanisms to viruses regardless of the induction time (Ton et al. 2009). ABA treatment decreases titers of Bamboo mosaic virus $(\mathrm{BaMV})$ in inoculated leaves of Arabidopsis (Alazem et al. 2014). In addition, several mutants defective in 


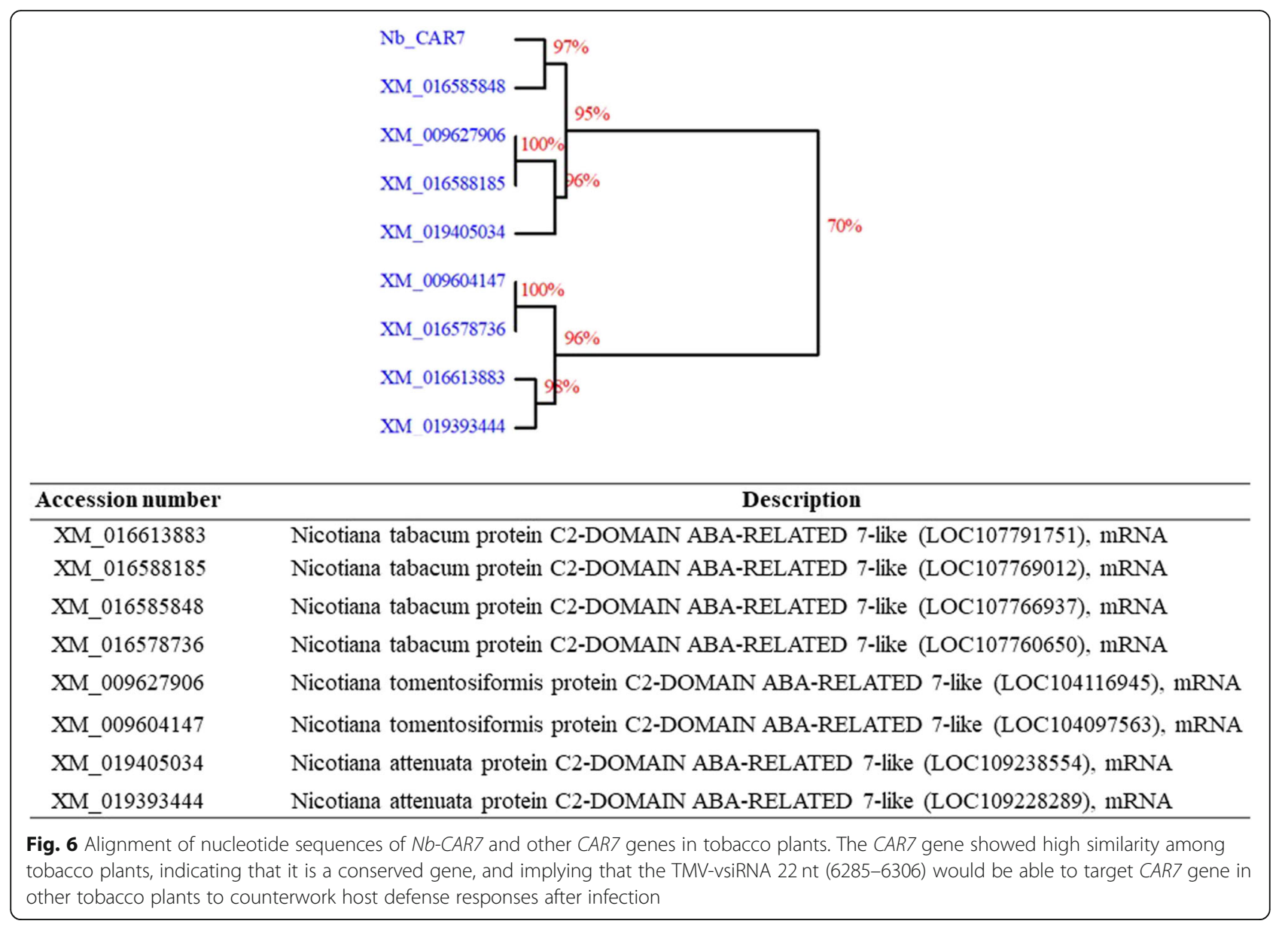

ABA signaling pathway exhibit enhanced accumulation of TMV crucifier strain in the upper systemically infected leaves of Arabidopsis but not in the inoculated leaves (Chen et al. 2013). There are a lot of reports about the increase of ABA content in virus-infected host plants, such as in CMV-infected N. benthamiana, BaMV-infected $A$. thaliana and $N$. benthamiana plants (Alazem et al. 2014), and in TMV-infected N. tabacum plants (Fraser and Whenham 1989). However, virus infection does not always induce an increase in ABA content in plants. For example, Potato virus $Y$ does not induce an increase of ABA in resistant potato cultivar Sante but induced an increase of jasmonic acid (JA) within the first few hours after virus infection (Flis et al. 2005; Kovač et al. 2009). A resistant tomato cultivar that carries the $R$-gene Tm- 1 has a higher ABA content than a susceptible cultivar. However, the ABA content in the resistant tomato cultivar remains unchanged after TMV infection (Whenham et al. 1986; Baebler et al. 2014).

ABA has been linked to antiviral silencing pathway which interferes with virus accumulation (Alazem and Lin 2015; Alazem et al. 2017). It is also involved in the microRNA (miRNA) pathway and affects maturation and stability of miRNAs (Lu and Fedoroff 2000). Additionally, it induces callose deposition at plasmodesmata, limiting viral cell-to-cell movement (Iglesias and Meins Jr 2000). ABA induces resistance against BaMV through Argonaute proteins (AGO 2 and 3), indicating that ABA regulates the expression of several members of the AGO family, and this regulation partially contributes to ABAmediated resistance against viruses (Alazem et al. 2017). In this study, based on the high nucleotide sequence homology, the TMV-vsiRNA 22 nt (6285-6306) derived from the juxtaposition of UPD and TLS of TMV induced silencing of a host gene, $\mathrm{Nb}-\mathrm{CAR7}$, in the ABA signaling pathway. Downregulation of $N b-C A R 7$ enhanced TMV RNA accumulation. In addition, the TMVvsiRNA $22 \mathrm{nt}$ (6285-6306) that targets $\mathrm{Nb}$-CAR7 for degradation possessed an adenosine at its $5^{\prime}$ end. The AGO2 and AGO4 could be involved in this targeting as AGO2 and AGO4 preferentially recruit small RNAs with a 5 ' terminal adenosine (Mi et al. 2008).

Previous studies have shown that vsiRNA derived from a plant virus can target a host gene for regulation (Shi et al. 2016). Some siRNAs from satellite RNA of virus can also target host genes. For example, CMV Y-sat produces a 22 nt sequence that is complementary to tobacco magnesium protoporphyrin chelatase subunit I gene ChlI to disrupt chlorophyll biosynthesis, which results in 
chlorotic symptom (Shimura et al. 2011). Not only targeting host genes, the vsiRNA has also been reported to target host long non-coding RNA to affect symptom development (Yang et al. 2019). The vsiRNAs generated from viroids could guide degradation of host mRNAs (Navarro et al. 2012). Also, a small RNA derived from the conserved region of Potato spindle tuber viroid (PSTVd) silences tomato FRIGIDA-like protein 3 gene (FRL3) and leads to early flowering of its host plants (Adkar-Purushothama et al. 2018). All these studies showed that vsiRNA could target host genes and affect disease symptoms development. In this study, the TMV-vsiRNA derived from the juxtaposition of UPD and TLS of TMV targets a host C2-domain ABA-related gene after virus infection. The results provide a new link of TMV-specific vsiRNAs regulating host genes during virus infection. The transcript level of $\mathrm{Nb}$-CAR7 was significantly decreased in TMV-infected plants, as compared with that of mock-inoculated and TMV-43A-inoculated plants, in which the 22 nt vsiRNAs are absent. Our results showed that the 22-nt TMVvsiRNA regulates its target gene $N b$-CAR7 through mRNA degradation. Whether it also regulates this target gene through translation inhibition needs further study.

\section{Conclusions}

Our study discovered that TMV-vsiRNA could target a host gene $\mathrm{Nb}$-CAR7. Overexpression of $\mathrm{Nb}$-CAR7 led to decreased TMV RNA accumulation in the inoculated leaves and silencing of $\mathrm{Nb}$-CAR7 enhanced TMV replication and resulted in a higher viral RNA accumulation. Moreover, the expression of $\mathrm{Nb}-\mathrm{CAR7}$ was closely correlated with the expression levels of abscisic acid (ABA) responsive genes in plants, which indicated that the effect of this gene to TMV RNA accumulation in host plants may be mediated by ABA signaling pathway.

\section{Methods}

\section{Plant inoculation and RNA isolation}

Nicotiana benthamiana plants were grown in a plant growth room at $25^{\circ} \mathrm{C}$ under a $16 \mathrm{~h} \mathrm{light} / 8 \mathrm{~h}$ dark photoperiod. Four-week-old plants were inoculated with $2 \mu \mathrm{g}$ of in vitro transcribed RNAs of TMV and TMV-43A, respectively. Total RNA was isolated from infected plants using TRIzol reagent (Invitrogen), according to the manufacturer's instructions. The low molecular weight (LMW) RNA was isolated from infected leaves using polyethylene glycol (PEG) (An et al. 2013). After PEG precipitation, the supernatant was transferred to a new tube, and then 1/10 volume of $\mathrm{NaOAC}$ and two volumes of absolute ethanol were added. The mixture was incubated at $-20^{\circ} \mathrm{C}$ for $30 \mathrm{~min}$ and centrifuged at $12,000 \times \mathrm{g}$ for $10 \mathrm{~min}$ at $4{ }^{\circ} \mathrm{C}$. The pellet was washed twice with $70 \%$ ethanol, briefly air dried and dissolved in $20 \mu \mathrm{L}$ of DEPC-treated water.

\section{Northern blot hybridization}

Low molecular weight (LMW) RNA samples were boiled for $5 \mathrm{~min}$, then immediately chilled on ice for $5 \mathrm{~min}$. For each RNA sample, an equal volume of formamide loading buffer ( $98 \%$ formamide, $40 \mathrm{mM}$ EDTA, $0.05 \mathrm{mg} / \mathrm{mL}$ bromophenol blue, $0.05 \mathrm{mg} / \mathrm{mL}$ xylene cyanol FF) was added before loading. Treated samples were loaded onto denaturing $17 \%$ polyacrylamide gels and run at $200 \mathrm{~mA}$. RNA was electro-blotted onto Hybond- $\mathrm{N}^{+}$membrane (Amersham) in $0.5 \times$ TBE buffer, immobilized onto the membrane by UV cross-linking, and hybridized overnight at $42{ }^{\circ} \mathrm{C}$ with DIG-labelled oligonucleotide probe corresponding to TMV (6285-6306 nt). The hybridized membranes were washed three times with $2 \times$ SSC and $0.2 \% \mathrm{SDS}$ for $20 \mathrm{~min}$ per wash at $42{ }^{\circ} \mathrm{C}$ and visualized by CSPD ready-to-use kit (Sigma-Aldrich). U6 RNA was used as an internal control.

\section{Validation of $\mathrm{Nb}$-CAR7 cleavage by 5'-RLM-RACE}

5 '-RNA ligase-mediated rapid amplification of cDNA ends (5'-RLM-RACE) was performed to validate $N b$ CAR7 cleavage using the GeneRacer ${ }^{\mathrm{TM}}$ kit (Invitrogen) with the manufacturer's instructions modified as follows. Briefly, $100 \mathrm{ng}$ total RNA was directly ligated to the RNA linker without prior treatment. After phenol/ chloroform extraction and precipitation, first-strand cDNA was synthesized using Gene-Specific Primer (Additional file 1: Table S1). In first-round 5'-RACE reaction, $1 \mu \mathrm{L}$ of primer was used including the GeneRacer $5^{\prime}$ primer from the kit and synthesized Gene-Specific Primer (Additional file 1: Table S1) with cycling as described in the GeneRacer ${ }^{\text {tox }}$ kit manual: 1 cycle of $94{ }^{\circ} \mathrm{C}$ for $2 \mathrm{~min}$, then 5 cycles of $94{ }^{\circ} \mathrm{C}$ for $30 \mathrm{~s}$ and $72^{\circ} \mathrm{C}$ for 1 min, then 5 cycles of $94{ }^{\circ} \mathrm{C}$ for $30 \mathrm{~s}$ and $70{ }^{\circ} \mathrm{C}$ for $1 \mathrm{~min}$, then 20 cycles of $94^{\circ} \mathrm{C}$ for $30 \mathrm{~s}$ and $68^{\circ} \mathrm{C}$ for $1 \mathrm{~min}$.

For the second-round $5^{\prime}$-RACE reaction, $1 \mu \mathrm{L}$ of the first-round reaction and internal primers was used, which are GeneRacer ${ }^{\text {Tx }}$ Nested primer from the kit and the synthesized Gene-Specific-Nested primer (Additional file 1: Table S1) with cycling conditions mentioned above except for an extension time of $15 \mathrm{~s}$. For this reaction, $5 \mu \mathrm{L}$ of product was analyzed on agarose gel. The PCR amplified fragment of $\sim 500 \mathrm{bp}$ (Fig. 2d) was sequenced using an ABI3130XL sequencer with BigDye ${ }^{\mathrm{Tx}}$ Terminator 3.1 Ready Reaction Cycling kit (Applied biosystems, USA) to confirm the cleavage of host $N b-C A R 7$ gene.

\section{Construction of $\mathrm{Nb}$-CAR7 over-expression and silencing vectors}

The complete ORF of $\mathrm{Nb}$-CAR7 was inserted into pGreen vector with a GFP tag using primers $N b$-CAR7-EcoRI-F and $N b$-CAR7-BamHI-R to construct a $N b$-CAR7 overexpression vector termed as 35S-NbCAR7-GFP. The nucleotide sequence of $\mathrm{Nb}$-CAR7 (101-497 nt) was amplified 
and ligated into pTRV2 vector with primers $\mathrm{Nb}$ $C A R 7_{(101-497)}$-EcoRI-F and Nb-CAR7 $7_{(101-497)}$-BamHI-R to construct a $N b-C A R 7$ silencing vector. The primers sequences were listed in the Additional file 1: Table S1.

TRV-based expression system for gene silencing and agroinfiltration-based transient gene overexpression $\mathrm{Nb}$-CAR7 was silenced by TRV-based gene silencing system. For agroinfiltration, an equal volume of Agrobacteria containing pTRV1 or pTRV2:Nb-CAR7 was mixed and infiltrated into two opposite leaves close to the bottom of four-week-old $N$. benthamiana plants using a 1$\mathrm{mL}$ syringe. The agroinfiltration of pTRV1 with empty pTRV2 served as control group. In vitro transcribed TMV RNA $(2 \mu \mathrm{g})$ was inoculated to the infiltrated leave of $\mathrm{Nb}$ CAR7-silenced plants at 7 days post infiltration. Both inoculated and upper leaves were collected for detection of viral RNAs accumulation at $7 \mathrm{dpi}$.

For agroinfiltration-based transient gene overexpression of $\mathrm{Nb}$-CAR7, Agrobacteria containing 35S-NbCAR7-GFP was infiltrated into two opposite leaves close to the bottom of four-week-old $N$. benthamiana plants using a 1-mL syringe. In vitro transcribed TMV RNA $(2 \mu \mathrm{g})$ was inoculated to the infiltrated leave of plants at 3 days post infiltration. The inoculated leaves and upper leaves were collected at $7 \mathrm{dpi}$.

\section{Agrobacterium infiltration}

$N$. benthamiana plants were grown in plastic pots at $25^{\circ} \mathrm{C}$ in a growth room under a $16 \mathrm{~h}$ light $/ 8 \mathrm{~h}$ dark cycle. Related TRV-gene-silencing vectors and overexpression vectors were introduced into Agrobacterium strain GV3101 by electroporation (BIO-RAD, Hercules, CA, USA), respectively. A 5-mL culture was grown overnight at $28{ }^{\circ} \mathrm{C}$ in the appropriate antibiotic selection medium. The next day, the culture was inoculated into a $50 \mathrm{~mL} \mathrm{LB}$ medium containing antibiotics, $10 \mathrm{mM}$ MES and $20 \mu \mathrm{M}$ acetosyringone. The culture was grown overnight in an incubator shaker at $28^{\circ} \mathrm{C}$. Agrobacterium cells were harvested and resuspended in infiltration media $(10 \mathrm{mM}$ $\mathrm{MgCl}_{2}, 10 \mathrm{mM} \mathrm{MES}, 200 \mu \mathrm{M}$ acetosyringone), adjusted to an OD of 2.0 and left at room temperature for $3 \mathrm{~h}$. Agrobacterium was infiltrated using a $1-\mathrm{mL}$ syringe.

\section{Quantitative RT-PCR (qRT-PCR) analysis}

To detect the expression level of $\mathrm{Nb}-\mathrm{CAR7}$ in plants, total RNA was isolated from mock-inoculated, TMVinfected, and TMV-43A-infected plants, respectively, at 4 dpi. cDNA was synthesized using Superscript III $^{\mathrm{mm}}$ First-Strand Synthesis System (Invitrogen, Carlsbad, $\mathrm{CA})$. The complete open reading frame (ORF) of $\mathrm{Nb}$ CAR7 was amplified using KOD-plus-Neo DNA polymerase (Toyobo, Tokyo, Japan) with primers $\mathrm{Nb}$ CAR7-F and Nb-CAR7-R.
For transient over-expression analysis, total RNA extracted from the 35S-NbCAR7-GFP-infiltrated leaves at 3 days post infiltration was used to determine the expression level of $\mathrm{Nb}$-CAR7. qRT-PCR was performed in triplicates with primers $N b-C A R 7-\mathrm{qF}$ and $N b-C A R 7-\mathrm{qR}$. Actin gene was used as an internal control with primers Actin-qF and Actin-qR.

For the TRV-induced gene silencing analysis, the ORF of $\mathrm{Nb}-\mathrm{CAR7}$ was amplified from total RNA of infiltrated and upper leaves at 7 days post infiltration, respectively. RT-PCR was performed using Superscript III ${ }^{\mathrm{TM}}$ FirstStrand Synthesis System (Invitrogen, Carlsbad, CA) and KOD-plus-Neo DNA polymerase (Toyobo, Tokyo, Japan) with primers $N b-C A R 7-\mathrm{F}$ and $N b-C A R 7-\mathrm{R}$.

To quantify TMV RNA accumulation in inoculated plants, total RNA isolated from inoculated leaves and upper leaves were extracted, respectively. Reverse transcription was performed using Superscript III Reverse Transcripatse kit (Life Technologies, Invitrogen) with primer TMV-R. qRT-PCR was performed in triplicates with primers TMV-qF and TMV-qR. To quantify the transcript level of LTI65 in transiently $\mathrm{Nb}$-CAR7-overexpressed leaves and $N b$-CAR7-silenced plants, primers $N b$-LTI65-F and Nb-LTI65-R were used to amplify the ORF of $N b$-LTI65. qRT-PCR was performed in triplicates with primers $N b-L T I 65-\mathrm{qF}$ and $N b$-LTI65-qR. $N$. benthamiana actin was used as an internal control.

All qRT-PCR analysis was performed using KAPA SYBR $^{\bullet}$ FAST qPCR Master Mix (2X) Kit (KAPA Biosystem, Wilmington, MA, USA) on the CFX384 Real-Time PCR system (Bio-Rad). Each $5 \mu \mathrm{L}$-reaction mix contained $20 \mathrm{ng}$ of cDNA, $200 \mathrm{nM}$ of each pair of target primers and $2.5 \mu \mathrm{L}$ of $2 \times$ SYBR Green PCR Master Mix. PCR conditions were as follows: $95^{\circ} \mathrm{C}$ for $3 \mathrm{~min}$, followed by 40 cycles of $95^{\circ} \mathrm{C}$ for $10 \mathrm{~s}$ and $60^{\circ} \mathrm{C}$ for $30 \mathrm{~s}$. All primers sequences used for $\mathrm{qRT}-\mathrm{PCR}$ reactions were listed in Additional file 1: Table S1.

\section{Statistical analysis}

All qRT-PCR expression assays were performed with three technical replicates from three independent biological replicates and repeated at least two times. The expression levels of $N b$-CAR7 and Nb-LTI65, and TMV viral RNAs accumulation level were normalized to that of actin gene, respectively. Data are expressed as mean \pm standard error. The statistical data analysis was conducted via unpaired $t$ test. A difference was considered to be significant when $P<0.05$ compared with control group.

\section{Supplementary information}

Supplementary information accompanies this paper at https://doi.org/10. 1186/s42483-020-00058-7.

Additional file 1: Table S1. List of primer sequences used in this study. 


\section{Abbreviations}

ABA: Abscisic acid; CAR: C2-domain abscisic acid-related; dpi: Days post inoculation; LTI65: Low-temperature-induced ABA responsive gene; PCR: Polymerase chain reaction; PK: Pseudoknot; RNA: Ribonucleic acid; RT: Reverse transcription; TLS: tRNA-like structure; TMV: Tobacco mosaic virus; UPD: Upstream pseudoknot domain; vsiRNA: Viral small interfering RNA

\section{Acknowledgements}

Not applicable.

\section{Authors' contributions}

SG performed experiments, analyzed the results and drafted the manuscript. SMW supervised the research and edited the manuscript. The authors read and approved the final manuscript.

\section{Funding}

This research was funded by the Singapore Ministry of Education Tier 1 Academic Research grant R-154-000-B23-114 through National University of Singapore, Suzhou Science \& Technology Bureau grant SNG2018039 and National Natural Science Foundation of China grant 31872639

\section{Availability of data and materials}

Not applicable.

\section{Ethics approval and consent to participate}

Not applicable.

\section{Consent for publication}

Not applicable.

\section{Competing interests}

The authors declare that they have no competing interests.

\section{Author details}

${ }^{1}$ Department of Biological Sciences, National University of Singapore, Singapore 117543, Singapore. ${ }^{2}$ Temasek Life Sciences Laboratory, 1 Research Link, Singapore 117604, Singapore. ${ }^{3}$ National University of Singapore (Suzhou) Research Institute, Suzhou 215123, Jiangsu, China.

Received: 24 April 2020 Accepted: 17 June 2020

Published online: 23 June 2020

\section{References}

Adkar-Purushothama CR, Sano T, Perreault JP. Viroid-derived small RNA induces early flowering in tomato plants by RNA silencing. Mol Plant Pathol. 2018;19: 2446-58.

Alazem M, He M-H, Moffett P, Lin N-S. Abscisic acid induces resistance against bamboo mosaic virus through Argonaute 2 and 3. Plant Physiol. 2017;174: 339-55.

Alazem M, Lin K-Y, Lin N-S. The abscisic acid pathway has multifaceted effects on the accumulation of Bamboo mosaic virus. Mol Plant-Microbe Interact. 2014 27:177-89.

Alazem M, Lin N-S. Roles of plant hormones in the regulation of host-virus interactions: plant hormone-virus interrelations. Mol Plant Pathol. 2015;16: 529-40.

Alazem M, Lin N-S. Antiviral roles of abscisic acid in plants. Front Plant Sci. 2017;8: 1760.

An Z, Li Y, Xie L, Zhai Q, Huang H. A rapid and economical method for low molecular weight RNA isolation from a wide variety of plant species. Biosci Biotechnol Biochem. 2013;77:1599-601.

Anderson JP, Badruzsaufari E, Schenk PM, Manners JM, Desmond OJ, Ehlert C, et al. Antagonistic interaction between abscisic acid and jasmonate-ethylene signaling pathways modulates defense gene expression and disease resistance in Arabidopsis. Plant Cell. 2004;16:3460-79.

Baebler Š, Witek K, Petek M, Stare K, Tušek-Žnidarič M, Pompe-Novak M, et al. Salicylic acid is an indispensable component of the ny-1 resistance-genemediated response against potato virus $Y$ infection in potato. J Exp Bot. 2014;65:1095-109.

Bivalkar-Mehla S, Vakharia J, Mehla R, Abreha M, Kanwar JR, Tikoo A, et al. Viral RNA silencing suppressors (RSS): novel strategy of viruses to ablate the host RNA interference (RNAi) defense system. Virus Res. 2011;155:1-9.
Chen L, Yan Z, Xia Z, Cheng Y, Jiao Z, Sun B, et al. A violaxanthin deepoxidase interacts with a viral suppressor of RNA silencing to inhibit virus amplification. Plant Physiol. 2017;175:1774-94.

Chen L, Zhang L, Li D, Wang F, Yu D. WRKY8 transcription factor functions in the TMV-cg defense response by mediating both abscisic acid and ethylene signaling in Arabidopsis. Proc Natl Acad Sci U S A. 2013;110:E1963-71.

Corbin JA, Evans JH, Landgraf KE, Falke JJ. Mechanism of specific membrane targeting by $\mathrm{C} 2$ domains: localized pools of target lipids enhance $\mathrm{Ca}^{2+}$ affinity. Biochemistry. 2007:46:4322-36.

Dai X, Zhuang Z, Zhao PX. psRNATarget: a plant small RNA target analysis server (2017 release). Nucleic Acids Res. 2018;46:W49-54.

Diaz M, Sanchez-Barrena MJ, Gonzalez-Rubio JM, Rodriguez L, Fernandez D, Antoni $R$, et al. Calcium-dependent oligomerization of CAR proteins at cell membrane modulates ABA signaling. Proc Natl Acad Sci U S A. 2016;113: E396-405.

Eamens A, Wang M-B, Smith NA, Waterhouse PM. RNA silencing in plants: yesterday, today, and tomorrow. Plant Physiol. 2008;147:456-68.

Ellinger D, Naumann M, Falter C, Zwikowics C, Jamrow T, Manisseri C, et al. Elevated early callose deposition results in complete penetration resistance to powdery mildew in Arabidopsis. Plant Physiol. 2013;161:1433-44.

Flis B, Hennig J, Strzelczyk-Żyta D, Gebhardt C, Marczewski W. The Ry-f sto gene from Solanum stoloniferum for extreme resistant to Potato virus $Y$ maps to potato chromosome XII and is diagnosed by PCR marker GP122718 in PVY resistant potato cultivars. Mol Breed. 2005;15:95-101.

Fraser RSS, Whenham RJ. Abscisic acid metabolism in tomato plants infected with tobacco mosaic virus: relationships with growth, symptoms and the tm1 gene for TMV resistance. Physiol Mol Plant Pathol. 1989;34(3):215-26.

Gallie DR, Feder JN, Schimke RT, Walbot V. Functional analysis of the tobacco mosaic virus tRNA-like structure in cytoplasmic gene regulation. Nucleic Acids Res. 1991:19:5031-6.

Gallie DR, Walbot V. RNA pseudoknot domain of tobacco mosaic virus can functionally substitute for a poly(a) tail in plant and animal cells. Genes Dev. 1990:4:1149-57.

Guo Q, Liu Q, Smith NA, Liang G, Wang M-B. RNA silencing in plants: mechanisms, technologies and applications in horticultural crops. Curr Genomics. 2016:17:476-89.

Guo S, Wong S-M. Deep sequencing analysis reveals a TMV mutant with a poly(a) tract reduces host defense responses in Nicotiana benthamiana. Virus Res. 2017;239:126-35.

Guo S, Wong S-M. Disruption of a stem-loop structure located upstream of pseudoknot domain in Tobacco mosaic virus enhanced its infectivity and viral RNA accumulation. Virology. 2018;519:170-9.

Iglesias VA, Meins F Jr. Movement of plant viruses is delayed in a beta-1,3glucanase-deficient mutant showing a reduced plasmodesmatal size exclusion limit and enhanced callose deposition. Plant J. 2000;21:157-66.

Kovač M, Müller A, Milovanovič Jarh D, Milavec M, Düchting P, Ravnikar M. Multiple hormone analysis indicates involvement of jasmonate signalling in the early defence of potato to potato virus $Y^{\text {NTN }}$. Biol Plant. 2009;53:195-9.

Li F, Wang A. RNA decay is an antiviral defense in plants that is counteracted by viral RNA silencing suppressors. PLoS Pathog. 2018;14:e1007228.

Li Z, Zhang T, Huang X, Zhou G. Impact of two reoviruses and their coinfection on the rice RNAi system and vsiRNA production. Viruses. 2018;10(11):594.

Lu C, Fedoroff N. A mutation in the Arabidopsis HYL1 gene encoding a dsRNA binding protein affects responses to abscisic acid, auxin, and cytokinin. Plant Cell. 2000;12:2351-66.

Mauch-Mani B, Mauch F. The role of abscisic acid in plant-pathogen interactions. Curr Opin Plant Biol. 2005;8:409-14.

Mi S, Cai T, Hu Y, Chen Y, Hodges E, Ni F, et al. Sorting of small RNAs into Arabidopsis Argonaute complexes is directed by the $5^{\prime}$ terminal nucleotide. Cell. 2008;133:116-27.

Nakashima K, Fujita Y, Katsura K, Maruyama K, Narusaka Y, Seki M, et al.

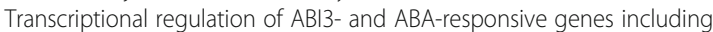
RD29B and RD29A in seeds, germinating embryos, and seedlings of Arabidopsis. Plant Mol Biol. 2006:60:51-68.

Navarro B, Gisel A, Rodio ME, Delgado S, Flores R, Di Serio F. Small RNAs containing the pathogenic determinant of a chloroplast-replicating viroid guide the degradation of a host mRNA as predicted by RNA silencing. Plant J. 2012;70:991-1003.

Niu S, Cao S, Huang L-J, Tan KC-L, Wong S-M. The length of an internal poly(a) tract of hibiscus latent Singapore virus is crucial for its replication. Virology. 2015;474:52-64. 
Osman TAM, Hemenway CL, Buck KW. Role of the 3' tRNA-like structure in tobacco mosaic virus minus-strand RNA synthesis by the viral RNAdependent RNA polymerase in vitro. J Virol. 2000;74:11671-80.

Pumplin N, Voinnet O. RNA silencing suppression by plant pathogens: defence, counter-defence and counter-counter-defence. Nat Rev Microbiol. 2013;11: $745-60$.

Rodriguez L, Gonzalez-Guzman M, Diaz M, Rodrigues A, Izquierdo-Garcia AC, Peirats-Llobet $\mathrm{M}$, et al. C2-domain abscisic acid-related proteins mediate the interaction of PYR/PYL/RCAR abscisic acid receptors with the plasma membrane and regulate abscisic acid sensitivity in Arabidopsis. Plant Cell. 2014;26:4802-20.

Sah SK, Reddy KR, Li J. Abscisic acid and abiotic stress tolerance in crop plants. Front Plant Sci. 2016;7:571.

Scholthof K-BG. Tobacco mosaic virus: a model system for plant biology. Annu Rev Phytopathol. 2004;42:13-34

Scholthof K-BG, Adkins S, Czosnek H, Palukaitis P, Jacquot E, Hohn T, et al. Top 10 plant viruses in molecular plant pathology. Mol Plant Pathol. 2011;12:938-54.

Shi B, Lin L, Wang S, Guo Q, Zhou H, Rong L, et al. Identification and regulation of host genes related to Rice stripe virus symptom production. New Phytol. 2016;209:1106-19.

Shimura H, Pantaleo V, Ishihara T, Myojo N, Inaba J, Sueda K, et al. A viral satellite RNA induces yellow symptoms on tobacco by targeting a gene involved in chlorophyll biosynthesis using the RNA silencing machinery. PLoS Pathog. 2011;7:e1002021.

Stahelin RV, Rafter JD, Das S, Cho W. The molecular basis of differential subcellular localization of $\mathrm{C} 2$ domains of protein kinase $\mathrm{C}$-alpha and group IVa cytosolic phospholipase A2. J Biol Chem. 2003;278:12452-60.

Takamatsu N, Watanabe Y, Meshi T, Okada Y. Mutational analysis of the pseudoknot region in the $3^{\prime}$ noncoding region of tobacco mosaic virus RNA. J Virol. 1990:64:3686-93.

Ton J, Flors V, Mauch-Mani B. The multifaceted role of ABA in disease resistance. Trends Plant Sci. 2009;14:310-7.

Uno Y, Furihata T, Abe H, Yoshida R, Shinozaki K, Yamaguchi-Shinozaki K. Arabidopsis basic leucine zipper transcription factors involved in an abscisic acid-dependent signal transduction pathway under drought and highsalinity conditions. Proc Natl Acad Sci U S A. 2000;97:11632-7.

Wang L-Y, Lin S-S, Hung T-H, Li T-K, Lin N-C, Shen T-L. Multiple domains of the tobacco mosaic virus p126 protein can independently suppress local and systemic RNA silencing. Mol Plant-Microbe Interact. 2012;25:648-57.

Whenham RJ, Fraser RSS, Brown LP, Payne JA. Tobacco-mosaic-virus-induced increase in abscisic-acid concentration in tobacco leaves: intracellular location in light and dark-green areas, and relationship to symptom development. Planta. 1986;168:592-8.

Yamaguchi-Shinozaki K, Shinozaki K. Arabidopsis DNA encoding two desiccationresponsive rd29 genes. Plant Physiol. 1993;101:1119-20.

Yamaguchi-Shinozaki K, Shinozaki K. A novel cis-acting element in an Arabidopsis gene is involved in responsiveness to drought, low-temperature, or high-salt stress. Plant Cell. 1994;6:251-64.

Yang Y, Liu T, Shen D, Wang J, Ling X, Hu Z, et al. Tomato yellow leaf curl virus intergenic siRNAs target a host long noncoding RNA to modulate disease symptoms. PLoS Pathog. 2019;15:e1007534.

Yasuda M, Ishikawa A, Jikumaru Y, Seki M, Umezawa T, Asami T, et al. Antagonistic interaction between systemic acquired resistance and the abscisic acid-mediated abiotic stress response in Arabidopsis. Plant Cell. 2008; 20:1678-92.

Zeenko W, Ryabova LA, Spirin AS, Rothnie HM, Hess D, Browning KS, et al. Eukaryotic elongation factor $1 \mathrm{~A}$ interacts with the upstream pseudoknot domain in the $3^{\prime}$ untranslated region of tobacco mosaic virus RNA. J Virol. 2002;76:5678-91.

Zhang X, Lai T, Zhang P, Zhang X, Yuan C, Jin Z, et al. Mini review: revisiting mobile RNA silencing in plants. Plant Sci. 2019;278:113-7.

Ready to submit your research? Choose BMC and benefit from:

- fast, convenient online submission

- thorough peer review by experienced researchers in your field

- rapid publication on acceptance

- support for research data, including large and complex data types

- gold Open Access which fosters wider collaboration and increased citations

- maximum visibility for your research: over $100 \mathrm{M}$ website views per year

At BMC, research is always in progress.

Learn more biomedcentral.com/submissions 